\title{
Anti-amoeba Effect of Branched Medium Chain Fatty Acids and Their Salts on Acanthamoeba Castellanii
}

\author{
Haruna Sasaki, Toshinari Koda, and Hiroshi Morita
}

\begin{abstract}
Acanthamoeba castellanii is an organism that causes Acanthamoeba keratitis, an infectious disease. Acanthamoeba are protists that generally inhabiting soils, freshwater, and sea water, but can also infect the cornea due to dirty water infiltration if contaminated contact lenses are worn. In this study, we focused on four branched medium chain fatty acids-2-ethylhexanoic acid, 3,5,5-trimethylhexanoic acid along with their salts, potassium 2-ethylhexanoate, and potassium 3,5,5-trimethylhexanoate. We examined their antimicrobial effect toward A. castellanii and aimed to search for new drugs to be used in contact lens disinfecting solutions. The minimum inhibitory concentrations (MICs) of 2-ethylhexanoic acid and 3,5,5-trimethylhexanoic acid showed growth reduction of 4-log units (99.99\% suppression) in $A$. castellanii upon 10 min incubation at $12.5 \mathrm{v} / \mathrm{v} \%$. In addition, at a diluted concentration of $6.25 \mathrm{v} / \mathrm{v} \%$, 2-ethylhexanoic acid produced a 4-log units reduction at $180 \mathrm{~min}$ and for 3,5,5-trimethylhexanoic acid, a 4-log units reduction at $60 \mathrm{~min}$. The MICs of potassium 2-ethylhexanoate showed growth reduction of $4 \mathrm{log}$ units in $A$. castellanii upon $10 \mathrm{~min}$ incubation at $2.50 \mathrm{w} / \mathrm{v} \%$. However, potassium 3,5,5-trimethylhexanoate showed growth reduction of 4-log units in $A$. castellanii upon 180 min incubation at 5.00 w/v\%. Based on the results of MICs, a verification test of 3,5,5-trimethylhexanoic acid and potassium 2-ethylhexanoate was conducted. As a result, approximately 2.1 logarithmic growths were observed in the control consisting of $10 \%$ Tween 80 compared to the initial cell number, and the amoeba growth reduction with 3,5,5-trimethylhexanoic acid could not be confirmed up to a concentration of $0.0122 \mathrm{v} / \mathrm{v} \%$. Potassium 2-ethylhexanoate salt was not confirmed to reduce amoeba growth up to $0.625 \mathrm{w} / \mathrm{v} \%$. However, the amoeba growth reduction could not be confirmed with the tests where control pH-adjusted solution was applied, either. In the light of the above-mentioned results, it was revealed that 3,5,5-trimethylhexanoic acid exhibits the highest anti-amoeba effect among the four tested branched medium chain fatty acids. This result indicated the possibility to use it in contact lens disinfectant solutions as a novel drug against Acanthamoeba keratitis.
\end{abstract}

Index Terms-2-ethylhexanoic acid, 3,5,5-trimethylhexanoic acid, acanthamoeba castellanii, Anti-amoeba effect.

\section{INTRODUCTION}

Acanthamoeba castellani is an organism that causes an infectious disease called Acanthamoeba keratitis, which was reported for the first time in the UK in 1974 [1] and in Japan

Manuscript received April 25, 2019; revised June 11, 2019.

H. Sasaki is with the Graduate School of Environmental Engineering, University of Kitakyushu, Fukuoka, Japan (e-mail: y7dab001@eng.kitakyu-u.ac.jp).

T. Koda is with the Nissan Chemical Corporation, Tokyo, Japan (e-mail: koda@nissanchem.co.jp).

H. Morita is with Faculty of Environment Engineering, University of Kitakyushu, Fukuoka, Japan (e-mail: morita@kitakyu-u.ac.jp). in 1988 [2] where over several hundred cases are presumed to occur currently [3]. In recent years, soft contact lenses mainly used by young people, showed an increasing trend [4], [5]. Acanthamoeba are protists generally found in soils, freshwater, and seawater [6], yet they can contaminate contact lenses leading to corneal infections due to dirty water infiltration [7]-[9]. Patients with Acanthamoeba keratitis may experience pain presenting with photophobia, ring-like stromal infiltrate, epithelial defects, and lid edema [10]. If Acanthamoeba keratitis is not treated adequately and aggressively, it can lead to loss of vision.

Currently, cold disinfection with hydrogen peroxide solution or multi-purpose solution (MPS) is commonly used for disinfecting contact lenses [11]-[13]. Particularly in the case with MPS; cleaning, rinsing, and disinfection of soft contact lenses can all be performed with the same liquid and it also serves as a preservative solution, making it a commonly used solution [11], [12]. However, the disinfection effect of MPS is weaker than other contact lens disinfection methods, and hypersensitivity may occur, pointing to another disadvantage [12], [13]. Moreover, according to the 2009 National Consumer Center report in Japan, it was shown that anti-amoebic activity of commercially available MPS is very low [14], [15]. MPS contains polyhexanide hydrochloride as a main component [14], [16], [17], however, even after an incubation period of 8 hours, commercially available MPS decreased only $1 / 10$ or less cells, in 4 of 8 strains [14], [15]. In addition, MPS is mainly targeted for eliminating bacteria and fungi, and is hardly considered for preventing eye infections due to amoeba.

Previous studies on anti-amoebic activity have reported antibacterial tests using straight chain fatty acids and their salts [15], in addition to antibacterial tests against fungi [18], bacteria [19] and other microbial agents. Fatty acid salts are carboxylic acid salts of chain hydrocarbons, and are surfactants formed from fatty acids and alkalis with both hydrophilic and hydrophobic properties. Even though fatty acids have no surface activity, as they are similar in structure to fatty acid salts, they have been used as comparative agents for fatty acid salts in previous studies.

However, an anti-amoeba study using branched medium chain fatty acids has not been reported so far. Therefore, in this study, we investigated anti-amoeba effects of branched medium chain fatty acids and their salts against $A$. castellanii. We focused on 2-ethylhexanoic acid and 3,5,5-trimethylhexanoic acid that are members of branched chain fatty acids. Branched chain fatty acids are characterized as colorless and transparent liquids without foreign matter, and are stable viscous liquids excellent in hydrophobicity, dispersibility, heat resistance, and low freezing point. Based 
on these characteristics, they are used in metal soaps, plasticizers, lubricants, surfactants, alkyd resins, fatty acid chlorides, and raw materials for cosmetics, and are excellent candidates for facilitating the exploration and discovery of new drugs. Based on these factors, the anti-amoeba effect of branched medium chain fatty acids and their salts against $A$. castellanii was investigated.

\section{METHOD}

\section{A. Acanthamoeba Strain and Growth Medium}

Acanthamoeba castellanii (ATCC 30010) was obtained from the ATCC (American Type Culture Collection, America), and Peptone-yeast-glucose medium (PYG, ATCC 712) was selected as the growth medium. Table I shows the medium composition. Basal medium, inorganic salt solution, and $2 \mathrm{M}$ glucose stock solution were adjusted to $\mathrm{pH} 6.5$ with $10 \% \mathrm{HCl}$ and $1 \mathrm{M} \mathrm{KOH}$.

TABLE I: PYG MEDIUM

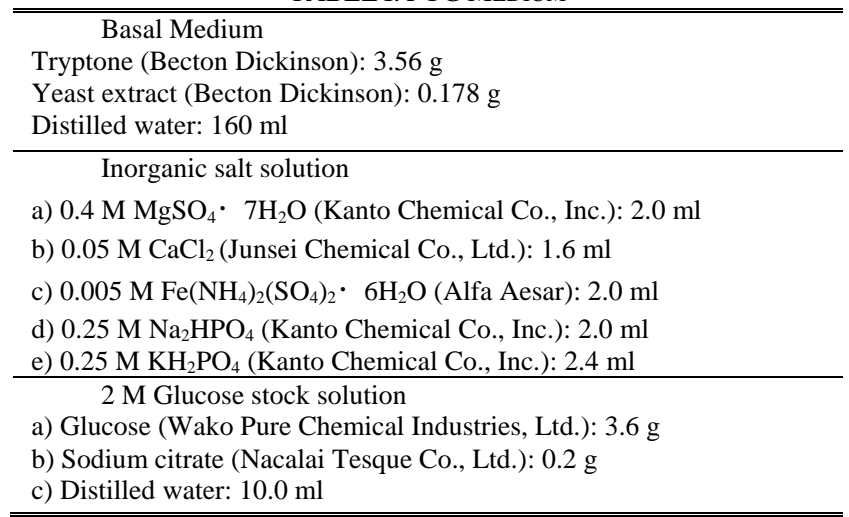

\section{B. Method of Trophozoite Cultivation}

The inorganic salt solution was autoclaved and added to an $300 \mathrm{ml}$ Erlenmeyer flask containing $160 \mathrm{ml}$ basal medium (Table I). Furthermore, $10 \mathrm{ml}$ of $2 \mathrm{M}$ glucose stock solution (Table I) was sterilized by filtration through a $0.25 \mu \mathrm{m}$ filter and added to the Erlenmeyer flask. Then, $45 \mathrm{ml}$ of the solution in the Erlenmeyer flask was dispensed into petri dishes $(\varphi 60 \times 45)$, and 10-fold diluted antibiotic, anti-mycotic solution (10000 units penicillin, $10 \mathrm{mg}$ streptomycin and $25 \mu \mathrm{g}$ amphotericin $\mathrm{B} / \mathrm{ml}$ ) was added per 5 $\mathrm{ml}$ of media. Finally, $500 \mu \mathrm{l}$ of $A$. castellanii cultivated with PYG medium $\left(3.0 \times 10^{5}\right.$ cells $\left./ \mathrm{ml}\right)$ was cultured on these dishes for 7 to 14 days at $30^{\circ} \mathrm{C}$.

TABLE II: STRUCTURE OF 2-ETHYLHEXANOIC ACID AND 3,5,5-TRIMETHYLHEXANOIC ACID

\begin{tabular}{|c|c|}
\hline Branched chain fatty acid & Structural formula \\
\hline 2-ethylhexanoic acid & \\
\hline 3,5,5-trimethylhexanoic acid & \\
\hline
\end{tabular}

\section{A. Fatty Acids and Their Salts}

Two branched chain fatty acids along with their salts were tested in this study. In this study, 2-ethylhexanoic acid and 3,5,5-trimethylhexanoic acid commercial products were tested. Their structural formulae are shown in Table II. For each of the two salts, those provided by Nissan Chemical Corporation were used. As for the concentration, a concentration already adjusted to $10 \%$ was used.

\section{B. Preparation of Cell Suspensions}

The number of trophozoites from the amoeba suspension produced by using the method described above, was assessed using an optical plastic plankton counter (Matsunami Glass Ind., Ltd., Osaka, Japan). The final amoeba suspension was adjusted to approximately $5.0 \times 10^{4}$ cells $/ \mathrm{ml}$.

\section{Assay of Anti-amoeba Activity}

A previously prepared and adjusted amoeba suspension of $1 \mathrm{ml}$ was desiccated, and $1 \mathrm{ml}$ of $10 \%$ Tween 80 was mixed with $1 \mathrm{ml}$ of the fatty acids with which the pellet was re-suspended. This mixture was incubated at $30^{\circ} \mathrm{C}$ and used as a control solution. In test solutions with fatty acid salt, a $\mathrm{pH}$-adjusted solution was used instead of Tween 80 .

Sampling was performed in $500 \mu \mathrm{l}$ aliquots at 10,60 , and $180 \mathrm{~min}$. The samples were added to microcentrifuge tubes containing $100 \mu \mathrm{l} \mathrm{Hanks'} \mathrm{Balanced} \mathrm{Salt} \mathrm{Solution} \mathrm{(Invitrogen)}$ and $400 \mu \mathrm{l} \quad 0.4 \%$ Trypan Blue Stain (Invitrogen), and incubated for $5 \mathrm{~min}$. Five-fold diluted samples were added to an optical plastic plankton counter and were allowed to stand for $5 \mathrm{~min}$. Due to osmotic pressure, the staining solution penetrates dead cells, which are thus stained blue. Therefore, the unstained viable cells were then counted using an optical microscope (ECLIPSE TS100; Nikon). The experiments were repeated at least thrice.

\section{Assay of Minimum Inhibitory Concentrations (MICs)}

$\mathrm{MIC}$ is the minimum concentration required to prevent the growth of microorganisms [20]. Thus, lower MIC values indicate greater drug efficacy. The MICs of fatty acids and fatty acid salts were measured to examine their anti-amoeba activity against $A$. castellanii and samples with the highest activity were determined.

Samples were prepared in 2-fold dilution series (final concentration of fatty acids: $12.5 \mathrm{v} / \mathrm{v} \%$, fatty acid salts: 1.25 $\mathrm{w} / \mathrm{v} \%$ ), and $400 \mu \mathrm{l}$ each of sample and amoeba suspension (final concentration of $5.0 \times 10^{4}$ cells $/ \mathrm{ml}$ ) was taken in a $1.5 \mathrm{ml}$ tube. After $3 \mathrm{~h}, 500 \mu \mathrm{l}$ of the sampling solution was transferred in a tube containing $100 \mu \mathrm{l}$ Hanks' Balanced Salt Solution and $400 \mu \mathrm{l}$ of $0.4 \%$ Trypan Blue Stain and stained for $5 \mathrm{~min} .300 \mu \mathrm{l}$ of the solution was taken onto an optical plastic plankton counter, to count the number of unstained viable cells using an optical microscope. These results indicated minimum concentrations at which no viable cells were observed, providing the MICs values of each fatty acids and their salts. The experiments were repeated at least thrice.

\section{E. Assessment with Verification Tests}

Results demonstrated a higher anti-amoeba activity against A. castellanii in 3,5,5-trimethylhexanoic acid and potassium 2-ethylhexanoate. Therefore, verification tests using contact lenses were conducted to evaluate the effectiveness of 3,5,5-trimethylhexanoic acid and potassium 
2-ethylhexanoate in MPS.

The selected amoeba for verification tests was the trophozoite of A. castellanii. The "seed 2 week pure" (Seed Co., Ltd.), a type of 2-week disposable soft contact lens was selected as the test lens.

The experiment was conducted according to the method described by Borazjani and Kilvington (2005) [21] and Kilvington et al. (1990) [22]. The amoeba suspension (1 ml) adjusted to $3.0 \times 10^{3}$ cells $/ \mathrm{ml}$ was added into 12 -well plates, and a soft contact lens was immersed and allowed to stand for $2 \mathrm{~h}$. Then, $2 \mathrm{ml}$ of the sample to be tested (10\% Tween 80 , 3,5,5-trimethylhexanoic acid and $\mathrm{pH}$-adjusted water, potassium 2-ethylhexanoate) was added in another plate and the soft contact lenses were transferred to these sample wells and incubated for $6 \mathrm{~h}$ for disinfection. The concentration of 3,5,5-trimethylhexanoic acid was $12.5 \mathrm{v} / \mathrm{v} \%$, and the concentration of potassium 2-ethylhexanoate was $1.25 \mathrm{w} / \mathrm{v} \%$. Then, the lenses were transferred to other wells containing 1 $\mathrm{ml}$ of the Acanthamoeba culture medium (PYG medium), detaching the amoeba adhering to the lenses in the wells. The number of viable cells was measured after 7 days of culture at $30^{\circ} \mathrm{C}$. An anti-amoebic effect was considered sufficient when the number of viable cells was less or equal to 10 cells $/ \mathrm{ml}$. The experiments were repeated at least three times.

\section{RESULTS}

\section{A. Effect of Fatty Acids and Their MICs on Acanthamoeba Castellanii}

Fig. 1 shows the time course of the anti-amoeba test of a 2-fold dilution series of the fatty acid, 2-ethylhexanoic acid, against $A$. castellanii.

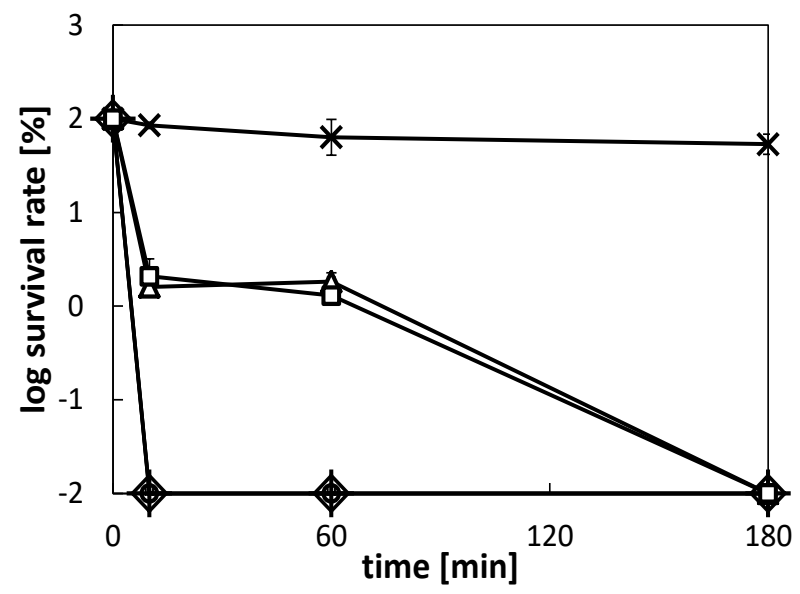

Fig. 1. Two-fold dilution series of 2-ethylhexanoic acid. The dots show the solutions: $10 \%$ tween 80 (control) $(\times), 2$-ethylhexanoic acid $50.0 \mathrm{v} / \mathrm{v} \%(\diamond)$,

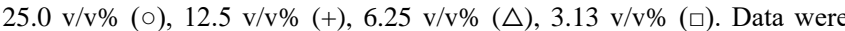
obtained from at least three independent experiments.

Fig. 2 shows the time course of the anti-amoeba test of a 2-fold dilution series of the fatty acid, 3,5,5-trimethylhexanoic acid, against $A$. castellanii. Figure 3 shows the microscopic images of the cells after $10 \mathrm{~min}$ of contact with the sample.

In Fig. 3 (A), vacuoles and similar structures were observed, but both (B) and (C) were stained dark blue and as the vacuole could not be observed, they were accepted as dead cells. The MICs of 2-ethylhexanoic acid and 3,5,5-trimethylhexanoic acid showed growth reduction of 4$\log$ units (99.99\% suppression) in A. castellanii upon $10 \mathrm{~min}$ incubation at $12.5 \mathrm{v} / \mathrm{v} \%$. However, $10 \%$ Tween 80 (control) had no effect. Therefore, it was demonstrated that both fatty acids showed anti-amoeba activity.

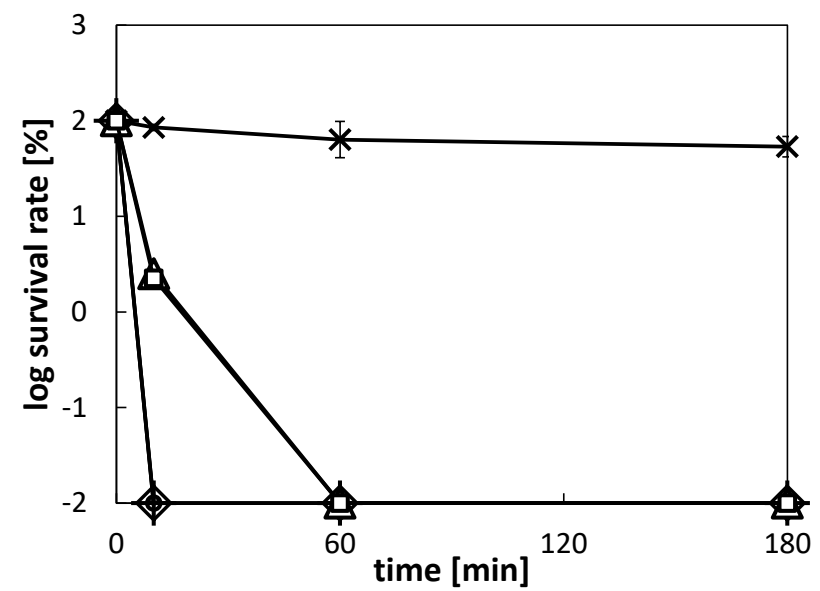

Fig. 2. Two-fold dilution series of 3,5,5-trimethylhexanoic acid. The dots show the solutions: $10 \%$ tween 80 (control) (x), 3,5,5-trimethylhexanoic acid $50.0 \mathrm{v} / \mathrm{v} \%(\diamond), 25.0 \mathrm{v} / \mathrm{v} \%(\circ), 12.5 \mathrm{v} / \mathrm{v} \%(+), 6.25 \mathrm{v} / \mathrm{v} \%(\triangle), 3.13 \mathrm{v} / \mathrm{v} \%$ $(\square)$. Data were obtained from at least three independent experiments.

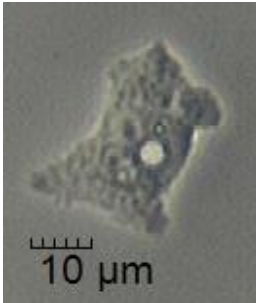

(A)

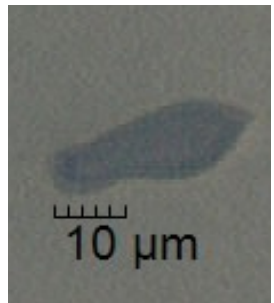

(B)

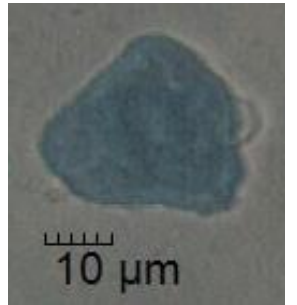

(C)
Fig. 3. Acanthamoeba cells after $180 \mathrm{~min}$ of contact with the control and fatty acids. (A) $10 \%$ Tween 80 , (B) 2-ethylhexanoic acid, (C) 3,5,5-trimethylhexanoic acid.

In addition, at a diluted concentration of $6.25 \mathrm{v} / \mathrm{v} \%$, 2-ethylhexanoic acid produced a 4-log units reduction at 180 min and 3,5,5-trimethylhexanoic acid a 4-log unit reduction at $60 \mathrm{~min}$.

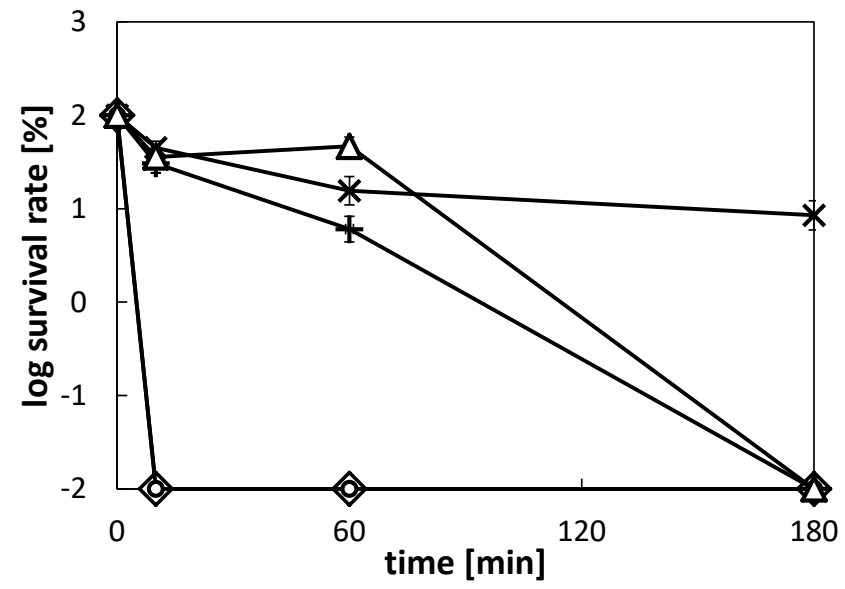

Fig. 4. Two-fold dilution series of potassium 2-ethylhexanoate. The dots show the $\mathrm{pH}$-adjusted solution (control) $(\times)$, potassium 2-ethylhexanoate $5.00 \mathrm{w} / \mathrm{v} \%(\diamond), 2.50 \mathrm{w} / \mathrm{v} \%(\circ), 1.25 \mathrm{w} / \mathrm{v} \%(+), 0.625 \mathrm{w} / \mathrm{v} \%(\triangle), 0.313$ $\mathrm{w} / \mathrm{v} \%(\square)$. Data were obtained from at least three independent experiments.

\section{A. Effect of Fatty Acid Salts and Their MICs on Acanthamoeba Castellanii}

Fig. 4 shows the time course of the anti-amoeba test of a 
2-fold dilution series of fatty acid salt, potassium 2-ethylhexanoic acid, against $A$. castellanii. Fig. 5 shows the time course of the anti-amoeba test of a 2-fold dilution series of fatty acid salt, potassium 3,5,5-trimethylhexanoic acid, against $A$. castellanii.

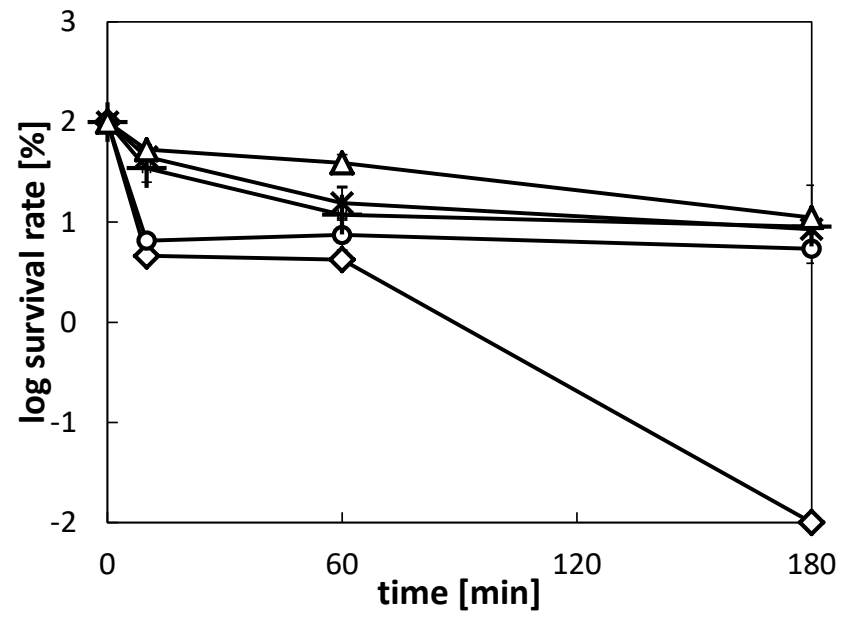

Fig. 5. Two-fold dilution series of potassium 3,5,5-trimethylhexanoate. The dots show the $\mathrm{pH}$-adjusted solution (control) ( $\times$ ), potassium 3,5,5-trimethylhexanoate $5.00 \mathrm{w} / \mathrm{v} \%(\diamond), 2.50 \mathrm{w} / \mathrm{v} \%(\circ), 1.25 \mathrm{w} / \mathrm{v} \%(+)$, $0.625 \mathrm{w} / \mathrm{v} \%(\triangle), 0.313 \mathrm{w} / \mathrm{v} \%(\square)$. Data were obtained from at least three independent experiments.

In Fig. 6 (A), as in Fig. 3, vacuole and similar structures were observed, but both (B) and (C) were stained black and the vacuole could not be observed. The MICs of potassium 2-ethylhexanoate showed growth reduction of $4 \log$ units in $A$ castellanii upon $10 \mathrm{~min}$ incubation at $2.50 \mathrm{w} / \mathrm{v} \%$. However, potassium 3,5,5-trimethylhexanoate showed growth reduction of $4 \mathrm{log}$ units in $A$. castellanii upon $180 \mathrm{~min}$ incubation at $5.00 \mathrm{w} / \mathrm{v} \%$. The $\mathrm{pH}$-adjusted solution (control) had no effect. Figure 6 shows the microscopic images of the cells after $180 \mathrm{~min}$ of contact with the sample.

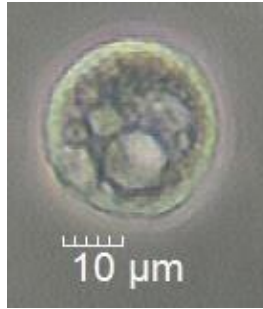

(A)

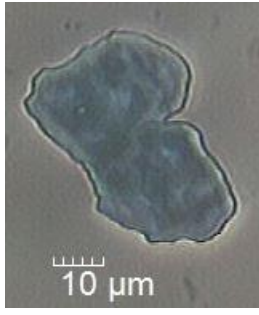

(B)
(C)

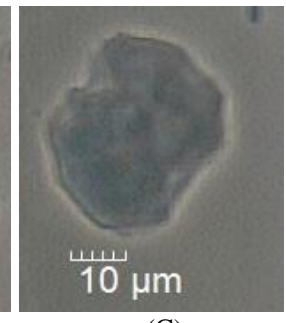

Fig. 6. Acanthamoeba cells after $180 \mathrm{~min}$ of contact with the fatty acid salts.

(A) $\mathrm{pH}$-adjusted solution (control), (B) potassium 2-ethylhexanoate, (C) potassium 3,5,5-trimethylhexanoate.

\section{B. Assessment of Verification Tests}

Fig. 7 shows the results of verification tests using the 3,5,5-trimethylhexanoic acid. About 2.1 logarithmic growths were observed in the control consisting of $10 \%$ Tween 80 when compared to the initial cell number, but 3,5,5-trimethylhexanoic acid's anti-amoeba effect could not be confirmed up to $0.0122 \mathrm{v} / \mathrm{v} \%$.

Fig. 8 shows the results of verification tests using the potassium 2-ethylhexanoate. Potassium 2-ethylhexanoate salt could not confirm eliminating amoeba up to $0.625 \mathrm{w} / \mathrm{v} \%$. However, the anti-amoeba effect could not be confirmed with the control $\mathrm{pH}$-adjusted solution.

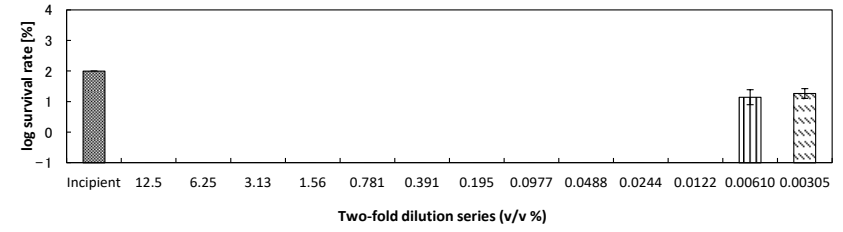

Fig. 7. 3, 5, 5-trimethylhexanoic acid verification experiment (two-fold dilution series from MIC value).

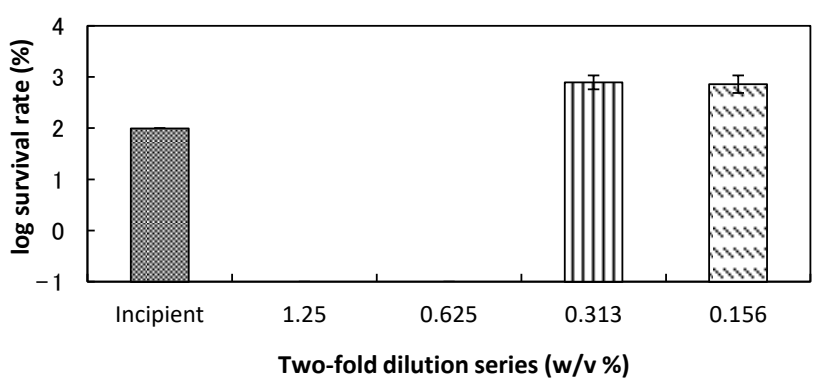

Fig. 8. Potassium 2-ethylhexanoate verification experiment (two-fold dilution series from MIC value)

\section{DISCUSSION}

The results of this study suggest that the branched medium chain fatty acids have higher anti-amoeba effect on amoebae than their potassium salts. Previous anti-amoeba studies on $A$. castellanii showed a 4-log reduction in growth when treated with $175 \mathrm{mM}$ potassium caprylate $(\mathrm{C} 8 \mathrm{~K})$, potassium caprate $(\mathrm{C} 10 \mathrm{~K})$, and potassium laurate $(\mathrm{C} 12 \mathrm{~K})$ for $180 \mathrm{~min}$ [15]. In addition, cell membrane disruption was observed after mixing the amoeba suspension with $\mathrm{C} 10 \mathrm{~K}$ or $\mathrm{C} 12 \mathrm{~K}$, and the $\mathrm{MIC}$ of $\mathrm{C} 10 \mathrm{~K}$ and $\mathrm{C} 12 \mathrm{~K}$ was $2.7 \mathrm{mM}$. Furthermore, in verification tests, both samples showed an anti-amoeba effect at a concentration of $5.5 \mathrm{mM}$.

In order to calculate the molarity of the sample used in this study, the weight per $1 \mathrm{~g}$ of the sample was measured three times, and the molarity was obtained using the average value and the molecular weight of the sample. As a result, the MIC of the anti-amoeba test in this study for 3,5,5-trimethylhexanoic acid (12.5 v/v\%) was $705 \mathrm{mM}$, and for potassium 2-ethylhexanoate $(2.50 \mathrm{w} / \mathrm{v} \%)$ was $139 \mathrm{mM}$. MIC in the verification tests was $0.689 \mathrm{mM}$ for 3,5,5-trimethylhexanoic acid $(0.0122 \mathrm{v} / \mathrm{v} \%)$ and $34.8 \mathrm{mM}$ for potassium 2-ethylhexanoate( $0.625 \mathrm{w} / \mathrm{v} \%$ ).

Contrary to previous ones, in this study, dead cells were confirmed even though the amoeba cell membrane was not ruptured, as shown in Figures 3 and 6. It was difficult to compare our anti-amoeba tests to others because previous studies conducted anti-amoeba tests for $180 \mathrm{~min}$. However, in the verification tests, 3,5,5-trimethylhexanoic acid was found to have an anti-amoeba effect at a concentration of about $1 / 8$ of the concentrations of $\mathrm{C} 10 \mathrm{~K}$ and $\mathrm{C} 12 \mathrm{~K}$ from the previous study. From these result, it can be deduced that the anti-amoeba effect of 3,5,5-trimethylhexanoic acid is higher than that of $\mathrm{C} 10 \mathrm{~K}$ and $\mathrm{C} 12 \mathrm{~K}$ from the previous study.

Petschow et al. showed the susceptibility of Helicobacter pylori to the bactericidal properties of medium chain monoglycerides and free fatty acids, which may also be a possible model for the mechanism of amoeba cell death [23]. They showed medium chain fatty acids, long chain fatty acids, and unsaturated fatty acids bind to the carrier of the electron transport chain while passing through the outer membrane, 
enabling their entry to the cell and cause disruption of the electron transport [23]. This is thought to be due to inhibition of FabI protein, which is a part of the lipid metabolism [24]. In a study by Watanabe [25] on an antimicrobial surfactant, the anionic surfactant was found to adhere to the surface of bacteria; the adsorbed surfactant exhibits affinity to the cell surface and destroys the cell membrane suggesting permeation through the polyprotein membrane. Therefore, it is thought that this resulted in an antibacterial effect.

\section{CONCLUSION}

Considering the results of this study, we examined the applicability of potassium 2-ethylhexanoate and 3,5,5-trimethylhexanoic acids as agents in contact lens disinfecting solutions. As a result, it was revealed through the verification tests that 3,5,5-trimethylhexanoic acid had an anti-amoeba effect at $0.689 \mathrm{mM}$ and potassium 2-ethylhexanoate at $34.8 \mathrm{mM}$. However, potassium 2-ethylhexanoate had a high $\mathrm{pH}$, thus it was not possible to clarify the effect of the sample itself in the verification tests. Furthermore, both potassium 2-ethylhexanoate and 3,5,5-trimethylhexanoic acids are considered to cause skin and eye irritations, and as they would directly contact the eyes through soft contact lenses soaked in a disinfectant solution containing these fatty acids, they do not seem applicable. Therefore, this study suggests that it can be used as a disinfectant for hard contact lenses that can be placed to the eyes after rinsing with tap water, once they are disinfected with 3,5,5-trimethylhexanoic acid.

\section{REFERENCES}

[1] J. Naginton, P. G. Watson, T. J. Playfair, J. Mcgill, B. R. Jones, and A D. McG. Steele, "Amoebic infection of the eye," The LANCET, vol. 2 , pp. 1537-1540, December 1974.

[2] Y. Ishibashi, Y. Matsumoto, R. Watanabe, S. Hommura, K. Yasuraoka, K. Ishii, et al., "Case of Acanthamoeba kerariris," Journal of Japanese Ophthalmological Society, vol. 92, no. 6, pp. 963-72, January 1988.

[3] M. Tokoro, T. Yamaguchi, M. Tanaka, A. Kobayashi, and Y. Oikawa, "Current status of molecular taxonomy in pathogenic protozoan parasites," Journal of the Jûzen Medical Society, vol. 117, no. 2, pp. 53-57, June 2008.

[4] K. Shinoda, A. Kobayashi, S. Miwa, A. Maeda, M. Sakurai, Y. Shirao, et al., "A case of non-Acanthamoebic keratitis with radial keratoneuritis," Folia Ophthalmologica Japonica, vol. 53, no. 11, pp. 894-897, November 2002.

[5] Study group of national surveillance of infectious keratitis in Japan, "National surveillance of infectious keratitis in Japan: Current status of isolates, patient background, and treatment," Journal of Japanese Ophthalmological Society, vol. 110, no. 12, pp. 961-971, December 2006.

[6] K. Ishii, Amoeba Picture Book, Japan: Kanbara Publishing Co., Ltd., 1992, ch. 1, pp. 216.

[7] A. B. Zimmerman, K. Richdale, G. L. Mitchell, B. T. Kinoshita, D. Y. Lam, H. Wagner, et al., "Water exposure is a common risk behavior among soft and gas-permeable contact lens wearers," Cornea, vol. 36, pp. 995-1001, August 2017.

[8] H. Yamaura, R. Shirasaka, K Matsumoto, T. Nakai, H. Horikami, and K. Ishii, "Acanthamoeba in house dust," Jpn. J. Parasitol, vol. 42, no. 2, pp. 130-133, April 1993.

[9] T. Miyazaki, K. Yagita, T. Yanagi, H. Uemura, and H. Kanbara, "Seasonal fluctuation of Acanthamoeba spp. contamination in water containers placed indoors and outdoors," Acta Med Nagasaki, vol. 52, pp. 13-18, February 2007.

[10] J. Lorenzo-Morales, N. A. Khan, and J. Walochnik, "An update on Acanthamoeba keratitis: Diagnosis, pathogenesis and treatment," Parasite, vol. 22, no. 10, pp. 1-20, February 2015.

[11] K. Hiti, J. Walochnik, E. M. Haller-Schober, C. Faschinger, and H. Aspöck, "Viability of Acanthamoeba after exposure to a multipurpose disinfecting contact lens solution and two hydrogen peroxide systems," British Journal of Ophthalmology, vol. 86, pp. 144-146, February 2002.

[12] N. Atkins, "Developments in lens care solution," The Optician, vol. 6037, no. 231, pp. 32-38, February 2006.

[13] H. Morishige, Y. Mano, H. Sasaoka, T. Nakamura, M. Tomita, K. Shiba, et al., "Disinfection of contact lens case and lens care solution by slightly acidic electrolyzed water," Japanese Journal of Environmental Infections, vol. 27, no. 1, pp. 13-19, October 2011.

[14] National Institute of National Consumer Affairs Center, "Disinfection performance against Acanthamoeba of soft contact lens disinfectants -Based on actual usage survey- (in Japanese)," pp. 1-32, December 2009.

[15] A. Tanaka, M. Era, Y. Obata, M. Masuda, T. Kawahara, T. Kanyama, et al., "Role of fatty acid salts as anti Acanthamoeba agents for disinfecting contact lens," Biocontrol Science, vol. 22, no. 3, pp. 153-161, February 2017.

[16] J. Santodomingo-Rubido, O. Mori, and S. Kawaminami, "Cytotoxicity and antimicrobial activity of six multipurpose soft contact lens disinfecting solutions," Ophthalmic Physiol Opt, vol. 26, pp. 476-482, November 2006.

[17] C. K. Choy, P. Cho, and M. V. Boost, "Cytotoxicity and effects on metabolism of contact lens care solutions on human corneal epithelium cells," Clinical and Experimental Optometry, vol. 95, pp. 198-206, March 2012.

[18] M. Era, S. Sakai, A. Tanaka, T. Kawahara, T. Kanyama, and H. Morita, "Antifungal activity of fatty acid salts against Penicillium pinophilum," Japan Journal of Food Engineering, vol. 16, no. 2, pp. 99-108, June 2015.

[19] M. Masuda, M. Era, T. Kawahara, T. Kanyama, and H. Morita, "Antibacterial effect of fatty acid salts on oral bacteria," Biocontrol Science, vol. 20, no. 3, pp. 209-213, March 2015.

[20] K. Takatori, "Mold inspection manual color atlas (in Japanese)," Techno System, pp. 214-215, Tokyo, 2009.

[21] R. N. Borazjani and S. Kilvington, "Efficacy of multipurpose solutions against Acanthamoeba species," Cont. Lens Anterior Eye, vol. 28, pp. 169-175, December 2005.

[22] S. Kilvington and D. F. P. Larkin, "Acanthamoeba adherence to contact lenses and removal by cleaning agents," Eye, vol. 4, no. 4, pp. 589-593, July 1990.

[23] B. W. Petschow, R. P. Batema, and L. L. Ford, "Susceptibility of Helicobacter pylori to bactericidal properties of medium-chain monoglycerides and free fatty acids," Antimicrobial Agents and Chemotherapy, vol. 40, no. 2, pp. 302-306. November 1996.

[24] C. J. Zheng, J. S. Yoo, T. G. Lee, H. Y. Cho, Y. H. Kim, and W. G. Kim, "Fatty acid synthesis is a target for antibacterial activity of unsaturated fatty acids," US National Library of Medicine National Institutes of Health, vol. 579, no. 23, pp. 5157-5162, 2005.

[25] S. Watanabe, "Present status and trends antimicrobial surface active agents," Oil Chemistry, vol. 29, no. 8, pp. 536-542, 1980.

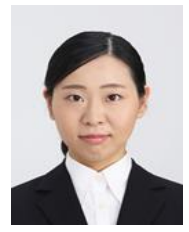

Haruna Sasaki is with the Graduate School of Environmental Engineering, University of Kitakyushu, Fukuoka, Japan from 2017 to the present

As research content, she is engaged in research on microbial control in indoor environment centering on environmental engineering.

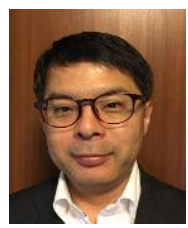

Toshinari Koda was born in Hyogo Prefecture, Japan and entered Nissan Chemical Corporation in 1991 after graduation from Faculty of Engineering, Nagoya Institude of Technology Aichi, Japan.

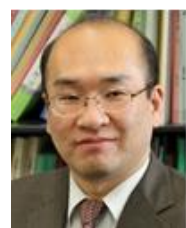

technology
Hiroshi Morita was born on September 20, 1970 in Nagoya, Japan. He received the B.E., M.E., and Ph.D. degrees in food science and technology from Kyushu University, Fukuoka, Japan, in 1994, 1996 and 1999 respectively. Presently he is a professor in Faculty of Environmental Engineering, The University of Kitakyushu, Japan. His research interests include biocontrol science, applied microbiology, and food 